\title{
Gluons, tadpoles, and color neutrality in a two-flavor color superconductor
}

\author{
Dennis D. Dietrich ${ }^{1}$ and Dirk H. Rischke ${ }^{2}$ \\ ${ }^{1}$ Laboratoire de Physique Théorique, Université Paris XI, Orsay, France \\ ${ }^{2}$ Institute for Theoretical Physics, University Frankfurt, Germany
}

October 5, 2018

\begin{abstract}
Considering cold, dense quark matter with two massless quark flavors, we demonstrate how, in a self-consistent calculation in the framework of QCD, the condensation of Cooper pairs induces a non-vanishing background color field. This background color field has precisely the right magnitude to cancel tadpole contributions and thus ensures overall color neutrality of the two-flavor color superconductor.
\end{abstract}

\section{Introduction and Conclusions}

Due to asymptotic freedom [1], at quark chemical potentials $\mu \gg \Lambda_{\mathrm{QCD}}$ single-gluon exchange is the dominant interaction between quarks in cold, dense quark matter. Single-gluon exchange is attractive in the color-antitriplet channel, which gives rise to the formation of quark Cooper pairs [2]. As this is analogous to what happens in ordinary superconductors [3], this phenomenon was termed color superconductivity [4, 5].

A well-studied color-superconducting system is cold, dense quark matter with two flavors of massless quarks [4, 5]. In this case, up and down quarks of, say, red and green color form anti-blue Cooper pairs with total spin zero in the color-antitriplet, flavor-singlet channel. Blue up and down quarks remain unpaired.

If there is a single chemical potential $\mu_{u}=\mu_{d} \equiv \mu$ for quark number, the system is color-neutral in the normal-conducting phase, and the number densities of red, green, and blue quarks are equal,

$$
n_{r}^{0}=n_{g}^{0}=n_{b}^{0} \equiv 2 N_{f} \int \frac{\mathrm{d}^{3} \mathbf{q}}{(2 \pi)^{3}} n_{\mathbf{q}}^{0}
$$

where $n_{\mathbf{q}}^{0} \equiv \Theta(\mu-q)$ is the occupation number for non-interacting, massless quarks. A natural question is then whether such a system is still color-neutral in the superconducting phase. Naively, one would think that this should be the case, as the total number of quarks does not change just because a part of them has formed Cooper pairs. However, at fixed quark chemical potential $\mu$ this is in fact not true, because pairing changes the dispersion relation for quasi-particle excitations [6],

$$
\epsilon_{\mathbf{q}}^{0} \equiv|\mu-q| \rightarrow \epsilon_{\mathbf{q}} \equiv \sqrt{(\mu-q)^{2}+\phi^{2}}
$$


where $\phi$ is the color-superconducting gap function. In turn, also the occupation number for quasiparticle excitations changes [6],

$$
n_{\mathbf{q}}^{0}=\Theta(\mu-q) \rightarrow n_{\mathbf{q}}=\frac{\epsilon_{\mathbf{q}}+\mu-q}{2 \epsilon_{\mathbf{q}}}
$$

It is not hard to see that, due to phase space $\mathrm{d}^{3} \mathbf{q} \sim \mathrm{d} q q^{2}$ and the symmetric "smearing" of the quasi-particle occupation number (3) around the Fermi surface, in a system with fixed quark chemical potential $\mu$ the total density of quasi-particle excitations corresponding to paired red and green quarks is larger than that for unpaired red, green, and blue quarks,

$$
n_{r}=n_{g} \equiv 2 N_{f} \int \frac{\mathrm{d}^{3} \mathbf{q}}{(2 \pi)^{3}} n_{\mathbf{q}}>n_{r}^{0}=n_{g}^{0}=n_{b}^{0} .
$$

This mismatch in color causes the system to be no longer color-neutral; it carries an excess of anti-blue (i.e., red and green in the color-antitriplet channel) over blue color charge. The integral in Eq. (44) appears to be ultraviolet divergent, but is actually not, because the gap function $\phi$ vanishes rapidly away from the Fermi surface, see also Sec. 4 .

It is a well-known fact that, after fixing the gauge, the formation of Cooper pairs in a two-flavor color superconductor spontaneously breaks $S U(3)_{c}$ to $S U(2)_{c}$. This remaining local gauge symmetry acts in the space of red and green quarks and is indeed preserved in the superconducting phase, because the number of red and green quasi-particle excitations is equal, cf. Eq. (44). However, according to the above argument, at fixed quark chemical potential $\mu$ the $S U(3)_{c}$ gauge symmetry appears also to be explicitly broken by the excess of anti-blue over blue color charge. Nevertheless, the system as a whole should still be color-neutral. The question then is how color neutrality can be restored. One possibility is to introduce a color-chemical potential $\mu_{8}$ "by hand", which reduces the Fermi surface for red and green quarks and enlarges the Fermi surface for blue quarks in a way to ensure color neutrality. This is the accepted procedure if one studies color superconductivity in the framework of Nambu-Jona-Lasinio-type models [7, 8]. In QCD, however, one would think that a more elegant solution is realized.

In Ref. [9], Gerhold and Rebhan argued that the inequality of red and green quasi-particle excitations compared to the number of unpaired blue quarks induces non-vanishing tadpole contributions, see Eq. (25) of Ref. [9]:

$$
\mathcal{T}_{a}^{\mu} \equiv \frac{2 g}{\pi^{2}} \mu \phi^{2} \ln \left(\frac{2 \mu}{\phi}\right)\left(T_{a}\right)_{33} g^{\mu 0}=-\sqrt{6} \mu \phi^{2} \delta_{a 8} g^{\mu 0} .
$$

Here, $g$ is the strong coupling constant and $\left(T_{a}\right)_{33}$ is the (33)-component of the generator $T_{a}$ of $S U(3)_{c}$. The last equality follows from the leading-order result $\phi \simeq 2 \mu \exp \left[-3 \pi^{2} /(\sqrt{2} g)\right]$ for the colorsuperconducting gap parameter [10] and from $\left(T_{a}\right)_{33} \equiv-\delta_{a 8} / \sqrt{3}$. (For the definition of the tadpole $\mathcal{T}_{a}^{\mu}$, see Ref. [9], Eq. (15), or Eq. (27) below.) It was argued in Ref. 99 that this tadpole contribution gives rise to an effective color-chemical potential associated with the eighth generator $T_{8}$ of $S U(3)_{c}$,

$$
\mu_{8}=-g \frac{1}{3 m_{g}^{2}} \sqrt{6} \mu \phi^{2}=-\sqrt{6} \pi^{2} \frac{\phi^{2}}{g \mu},
$$

where $m_{g} \equiv g \mu /(\sqrt{2} \pi)$ is the gluon mass parameter in cold, dense quark matter with $N_{f}=2$ flavors of massless quarks. The prefactor arises from attaching a static, electric gluon with propagator $1 /\left(3 m_{g}^{2}\right)$ to the tadpole (5), with coupling constant $g$. The resulting diagram then affects the quark propagator in the manner of a chemical potential. Moreover, Gerhold and Rebhan concluded that the total system is color-neutral, because in a gauge which does not involve the zeroth component of the gauge field, $A_{0}^{a}$, the generating functional contains a functional integral $\int \mathcal{D} A_{0}^{a} \exp \left[i \int_{X} A_{0}^{a} N_{a}\right] \equiv \delta\left[N_{a}\right]$, where $N_{a}$ is the density of adjoint color charge $a$. The $\delta$-functional then ensures that $N_{a} \equiv 0$. 
The present paper can be considered as an addendum to Ref. [9] and is motivated by the following argument. In general, superconductivity is a non-perturbative phenomenon. A perturbative calculation will never produce a gap in the quasi-particle excitation spectrum; the gap parameter has to be determined from a self-consistent solution (possibly within a certain many-body approximation scheme) of a Dyson-Schwinger equation for the quark two-point function [3]. (Usually, one employs the mean-field approximation to compute the gap, for more details, see Refs. [5, 8] or Secs. 2 and 3 below.) However, if the gap is computed in a self-consistent manner, then one should also incorporate the color-chemical potential (6) in a self-consistent way.

This is done in the present paper. Before we go into the details of the calculation, let us briefly state our conclusions. We shall indeed obtain an effective chemical potential $\mu_{8}$, which precisely assumes the value (6). In a self-consistent calculation, this chemical potential does not arise from a nonvanishing tadpole (5), but from a non-vanishing expectation value of the gluon field, $A_{\mu}^{a} \equiv A g_{\mu 0} \delta^{a 8}$, with $A=$ const.. This background field is a self-consistent solution of the Yang-Mills equation and acts like an effective chemical potential, $\mu_{8} \equiv g A$, in the quark propagator. The possibility of a non-vanishing expectation value for the gluon field was also mentioned in Ref. 9]. Then, computing the tadpole selfconsistently with this background field, we shall see that it has precisely the right magnitude to cancel the tadpole (5). This cancellation is of crucial importance, as the tadpole (5) is, up to a factor of $g$, just the color-charge density associated with the eighth direction in adjoint color space. The vanishing of the tadpole due to the non-zero color background field thus ensures color neutrality in the self-consistent calculation.

The remainder of this paper is organized as follows. In Sec. 2 we introduce the effective action for color-superconducting quark matter. To this end, it is most convenient to work in the framework of the Cornwall-Jackiw-Tomboulis (CJT) formalism [1]. In Sec. 3] we derive and discuss the stationarity conditions for the effective action which determine the expectation values for the one- and two-point functions of the theory. The former are the Dirac equation for the quark field and the Yang-Mills equation for the gluon field, while the latter represent Dyson-Schwinger equations for the quark and gluon propagator. In Sec. 4 we solve the Yang-Mills equation self-consistently and show that the solution is a non-zero expectation value for the gluon field which cancels the tadpole contribution. Finally, in Sec. 5 we demonstrate how this non-zero background field provides color neutrality in the color superconductor.

Our units are $\hbar=c=k_{B}=1$. Four-vectors are denoted by capital letters, $K^{\mu} \equiv\left(k_{0}, \mathbf{k}\right)$, where the three-vector $\mathbf{k}$ has modulus $k \equiv|\mathbf{k}|$ and direction $\hat{\mathbf{k}} \equiv \mathbf{k} / k$. We work in Euclidean space with imaginary time $\tau \equiv i t=i x_{0}$, but nevertheless adhere to a notation familiar from Minkowski space, i.e., $K^{\mu} \equiv\left(k_{0}, \mathbf{k}\right) \equiv g^{\mu \nu} K_{\nu}$, with the metric tensor $g^{\mu \nu}=\operatorname{diag}(+,-,-,-)$. Space-time integrals are defined as $\int_{X} \equiv \int_{0}^{1 / T} \mathrm{~d} \tau \int_{V} \mathrm{~d}^{3} \mathbf{x}$, while the four-dimensional $\delta$-function is $\delta^{(4)}(X-Y) \equiv-i \delta\left(x_{0}-y_{0}\right) \delta^{(3)}(\mathbf{x}-\mathbf{y})$. Integrals in momentum space are defined as $\int_{K} \equiv T \sum_{n} \int \mathrm{d}^{3} \mathbf{k} /(2 \pi)^{3}$, where the sum runs over the Matsubara frequencies $\omega_{n}^{\mathrm{b}}=2 n \pi T$ for bosons and $\omega_{n}^{\mathrm{f}}=(2 n+1) \pi T$ for fermions, $n=0, \pm 1, \pm 2, \ldots$.

\section{Effective action for color-superconducting quark matter}

In superconducting systems, it is advantageous to introduce charge-conjugate fermion degrees of freedom in addition to the "usual" fermions and work in the so-called Nambu-Gor'kov basis [3]. Quarks with $N_{c}$ colors and $N_{f}$ flavors are then described by the $8 N_{c} N_{f}$-component spinors [6]

$$
\Psi \equiv\left(\begin{array}{c}
\psi \\
\psi_{C}
\end{array}\right), \quad \bar{\Psi} \equiv\left(\bar{\psi}, \bar{\psi}_{C}\right)
$$

where $\psi$ is the usual $4 N_{c} N_{f^{-}}$component quark spinor, $\bar{\psi} \equiv \psi^{\dagger} \gamma_{0}$ the adjoint quark spinor, and $\psi_{C} \equiv$ $C \bar{\psi}^{T}$ the charge-conjugate quark spinor, with the adjoint $\bar{\psi}_{C} \equiv \psi^{T} C$. Here, $C \equiv i \gamma^{2} \gamma_{0}$ is the charge 
conjugation matrix, $C^{-1} \equiv C^{T} \equiv C^{\dagger} \equiv-C$. The QCD tree-level action reads [5]

$$
I[\bar{\Psi}, \Psi, A]=-\frac{1}{4} \int_{X} F_{a}^{\mu \nu}(X) F_{\mu \nu}^{a}(X)+\frac{1}{2} \int_{X, Y} \bar{\Psi}(X) S_{0}^{-1}(X, Y) \Psi(Y)
$$

where $F_{\mu \nu}^{a} \equiv \partial_{\mu} A_{\nu}^{a}-\partial_{\nu} A_{\mu}^{a}+g f^{a b c} A_{\mu}^{b} A_{\nu}^{c}$ is the gluon field strength tensor. The factor $1 / 2$ in front of the fermionic contribution compensates for the doubling of the degrees of freedom in the Nambu-Gor'kov basis (17). The inverse tree-level quark propagator in the presence of a gluon field $A_{\mu}^{a}$ and a quark number chemical potential $\mu$ is

$$
S_{0}^{-1}(X, Y) \equiv-2 \frac{\delta^{2} I[\bar{\Psi}, \Psi, A]}{\delta \bar{\Psi}(X) \delta \Psi(Y)}=\left(\begin{array}{cc}
i \gamma_{\mu} D_{X}^{\mu}+\mu \gamma_{0}-m & 0 \\
0 & i \gamma_{\mu} D_{C, X}^{\mu}-\mu \gamma_{0}-m
\end{array}\right) \delta^{(4)}(X-Y) .
$$

The dependence on the gluon field enters through the covariant derivative $D_{X}^{\mu} \equiv \partial_{X}^{\mu}-i g A_{a}^{\mu}(X) T_{a}$ and its charge-conjugate counter-part $D_{C, X}^{\mu} \equiv \partial_{X}^{\mu}+i g A_{a}^{\mu}(X) T_{a}^{T}$. As we shall show below, in a two-flavor color superconductor [5], $A_{\mu}^{a}$ assumes a classical expectation value $A_{\mu}^{a} \equiv A g_{\mu 0} \delta^{a 8}$. (An analogous conclusion for the so-called color-flavor-locked phase [12] was drawn in Ref. [13]). From Eq. (9) it is obvious that this expectation value assumes the role of a color-chemical potential $\mu_{8} \equiv g A$ associated with the color-charge generator $T_{8}[8$.

The manner in which Nambu-Gor'kov quark spinors couple to the gluon field in Eq. (9) suggests the following definition for the quark-gluon vertex in Nambu-Gor'kov space,

$$
\Gamma_{a}^{\mu}=\left(\begin{array}{cc}
\gamma^{\mu} T_{a} & 0 \\
0 & -\gamma^{\mu} T_{a}^{T}
\end{array}\right)
$$

such that the quark-gluon coupling in the action (8) can be simply written as $g A_{\mu}^{a} \bar{\Psi} \Gamma_{a}^{\mu} \Psi$.

The most convenient way to derive the gap equations for color-superconducting quark matter is within the framework of the CJT formalism [1] where the effective action of QCD takes the form [5, 8, 14 ]

$$
\begin{aligned}
\Gamma[\bar{\Psi}, \Psi, A, S, \Delta] & =I[\bar{\Psi}, \Psi, A]-\frac{1}{2} \operatorname{Tr} \ln \Delta^{-1}-\frac{1}{2} \operatorname{Tr}\left(\Delta_{0}^{-1} \Delta-1\right) \\
& +\frac{1}{2} \operatorname{Tr} \ln S^{-1}+\frac{1}{2} \operatorname{Tr}\left(S_{0}^{-1} S-1\right)+\Gamma_{2}[\bar{\Psi}, \Psi, A, S, \Delta]
\end{aligned}
$$

From now on, $\bar{\Psi}, \Psi, A$ denote the expectation values of the quark and gluon fields. The quantities $\Delta$ and $S$ are the full gluon and quark propagators, respectively. The inverse tree-level quark propagator $S_{0}^{-1}$ was already introduced in Eq. (9). Its gluonic counter-part $\Delta_{0}^{-1}$ can be computed from Eq. (8) via

$$
\Delta_{0}^{-1}{ }_{a b}^{\mu \nu}(X, Y) \equiv \frac{\delta^{2} I[\bar{\Psi}, \Psi, A]}{\delta A_{\mu}^{a}(X) \delta A_{\nu}^{b}(Y)}
$$

but we refrain from giving its explicit form, since it will not be required in the following. However, we note that, similar to the inverse tree-level quark propagator (92), also the inverse tree-level gluon propagator depends on the gluon field $A_{\mu}^{a}$.

The traces in Eq. (11) run over space-time, Nambu-Gor'kov, color, flavor, and Dirac indices. The functional $\Gamma_{2}$ is the sum of all two-particle irreducible (2PI) diagrams. It is impossible to evaluate all 2PI diagrams exactly. However, the advantage of the CJT effective action (11) is that truncating the sum $\Gamma_{2}$ after a finite number of terms still provides a well-defined many-body approximation. Here we only include the sunset-type diagram shown in Fig. 1 of Ref. [8], which leads to the so-called mean-field approximation,

$$
\Gamma_{2}=-\frac{g^{2}}{4} \int_{X, Y} \operatorname{Tr}\left[\Gamma_{a}^{\mu} S(X, Y) \Gamma_{b}^{\nu} S(Y, X)\right] \Delta_{\mu \nu}^{a b}(X, Y)
$$


where the trace now runs only over Nambu-Gor'kov, color, flavor, and Dirac indices, and $\Gamma_{a}^{\mu}, \Gamma_{b}^{\nu}$ are the Nambu-Gor'kov vertices introduced in Eq. (10).

So far, we have not discussed the question of gauge fixing. This can be done on the level of the inverse tree-level gluon propagator (12), where appropriate terms have to be added. One also has to include ghost degrees of freedom in the CJT effective action (11). In principle, these are necessary to cancel the contribution from the unphysical gluon degrees of freedom to the effective action, but at temperatures of relevance for color superconductivity, $T \sim \phi \sim \mu \exp (-1 / g) \ll \mu$ in weak coupling, they can be neglected [5].

\section{Stationarity conditions for the effective action}

The CJT formalism allows to compute the expectation values for one- and two-point functions of the theory from the stationarity conditions

$$
\begin{aligned}
0 & =\frac{\delta \Gamma}{\delta \bar{\Psi}}=\frac{\delta \Gamma}{\delta \Psi}, \\
0 & =\frac{\delta \Gamma}{\delta A}, \\
0 & =\frac{\delta \Gamma}{\delta S}, \\
0 & =\frac{\delta \Gamma}{\delta \Delta} .
\end{aligned}
$$

Performing the variation of $\Gamma$ with respect to $\bar{\Psi}$, Eq. (14) yields the Dirac equation for the NambuGor'kov quark spinor $\Psi$ in an external gluon field (correspondingly, the variation with respect to $\Psi$ yields the Dirac equation for the adjoint spinor $\bar{\Psi}$ ). We do not give this equation explicitly, as the solution has to be trivial, $\Psi \equiv 0$ (and, correspondingly, $\bar{\Psi}=0$ ), since Grassmann-valued fields cannot have a classical expectation value.

Equation (15) is the Yang-Mills equation for the gluon field,

$$
\mathcal{D}_{X \nu}^{a b} F_{b}^{\nu \mu}(X)=\frac{\delta}{\delta A_{\mu}^{a}(X)}\left[\frac{1}{2} \operatorname{Tr}\left(\Delta_{0}^{-1} \Delta-S_{0}^{-1} S\right)-\Gamma_{2}\right],
$$

where $\mathcal{D}_{X \nu}^{a b}=\partial_{X \nu} \delta^{a b}-g f^{a b c} A_{\nu}^{c}(X)$ is the covariant derivative in the adjoint representation. The first two terms on the right-hand side are the contributions from gluon and quark tadpoles. We should add a remark concerning our nomenclature. The first term involves a gluon loop, wherefore we choose to call it the "gluon tadpole" contribution, while the second involves a quark loop (also recognizable by the relative minus sign with respect to the gluon tadpole), wherefore we call it the "quark tadpole". This nomenclature is based on the "body" of the tadpole, which can consist of a gluon or a quark loop. It should not be confused with that of Ref. 9] where tadpoles are named according to their "tail", i.e., they are always referred to as "gluon" tadpoles. The functional derivative with respect to $A_{\mu}^{a}$ acting on the trace is non-trivial because of the dependence of the inverse tree-level propagators $\Delta_{0}^{-1}$ and $S_{0}^{-1}$ on the gluon field, cf. Eq. (9). The last term is non-zero, if $\Gamma_{2}$ contains $2 \mathrm{PI}$ diagrams with an explicit dependence on $A_{\mu}^{a}$. It vanishes in our approximation (13) for $\Gamma_{2}$. We solve the Yang-Mills equation (18) explicitly in Sec. 4 .

Equation (16) is a Dyson-Schwinger equation for the full quark propagator,

$$
S^{-1}(X, Y)=S_{0}^{-1}(X, Y)+\Sigma(X, Y),
$$

where the quark self-energy is defined as

$$
\Sigma(X, Y) \equiv 2 \frac{\delta \Gamma_{2}}{\delta S(Y, X)} .
$$


In the mean-field approximation (13) for $\Gamma_{2}$,

$$
\Sigma(X, Y)=-g^{2} \Gamma_{a}^{\mu} S(X, Y) \Gamma_{b}^{\nu} \Delta_{\mu \nu}^{a b}(X, Y) .
$$

Assuming translational invariance, this becomes the usual result in momentum space [5],

$$
\Sigma(K)=-g^{2} \int_{Q} \Gamma_{a}^{\mu} S(Q) \Gamma_{b}^{\nu} \Delta_{\mu \nu}^{a b}(K-Q) .
$$

As discussed in great detail in Ref. [5], the diagonal Nambu-Gor'kov components of this expression correspond to the one-loop self-energy for quarks and charge-conjugate quarks, respectively. The offdiagonal components correspond to the gap equation for the color-superconducting gap parameter, which has been solved in Ref. [10].

However, all previous solutions of the QCD gap equation have neglected the possibility that the full quark propagator $S$ may (implicitly) depend on a non-vanishing expectation value for the gluon field. This dependence of $S$ on $A_{\mu}^{a}$ enters via Eq. (19) through the dependence of the tree-level propagator $S_{0}^{-1}$, Eq. (9), on $A_{\mu}^{a}$. As will be shown below, the numerical value for the expectation value of the gluon field is of order $\phi^{2} /\left(g^{2} \mu\right)$. This is parametrically of higher order, such that the previous solution of the QCD gap equation, which was obtained to subleading order in the strong coupling constant $g$, remains correct. (For a definition of the term "subleading order" in the context of the QCD gap equation, see Ref. [5.) Nevertheless, the non-zero expectation value of the gluon field will prove to be important to ensure color neutrality in the color superconductor.

Finally, Eq. (17) is a Dyson-Schwinger equation for the gluon propagator,

$$
\Delta_{a b}^{-1^{\mu \nu}}(X, Y)=\Delta_{0}^{-1 \mu \nu}(X, Y)+\Pi_{a b}^{\mu \nu}(X, Y)
$$

The gluon self-energy is defined as

$$
\Pi_{a b}^{\mu \nu}(X, Y) \equiv-2 \frac{\delta \Gamma_{2}}{\delta \Delta_{\nu \mu}^{b a}(Y, X)},
$$

which, in the mean-field approximation (13), becomes

$$
\Pi_{a b}^{\mu \nu}(X, Y)=\frac{g^{2}}{2} \operatorname{Tr}\left[\Gamma_{a}^{\mu} S(X, Y) \Gamma_{b}^{\nu} S(Y, X)\right]
$$

where the trace runs over Nambu-Gor'kov, color, flavor, and Dirac indices. In momentum space, this expression reads

$$
\Pi_{a b}^{\mu \nu}(P)=\frac{g^{2}}{2} \int_{K} \operatorname{Tr}\left[\Gamma_{a}^{\mu} S(K) \Gamma_{b}^{\nu} S(K-P)\right] .
$$

For a two-flavor color superconductor, the gluon self-energy has been computed as a function of energy $p_{0}$ and momentum $\mathbf{p}$ in Ref. [15]. For other color-superconducting phases, only the zero-energy, lowmomentum limit of the gluon self-energy is known, from which one deduces the Debye and Meissner masses. In the color-flavor-locked phase [12 these were computed in Ref. [16]. In the polar and colorspin-locked phases [17, where each quark flavor pairs at its own Fermi surface and Cooper pairs carry total spin one, the Debye and Meissner masses have been calculated in Ref. [18].

\section{Solution of the Yang-Mills equation}

In the following, we shall show that, in a two-flavor color superconductor, the solution of the YangMills equation (18) is a constant background field $A_{\mu}^{a} \equiv A g_{\mu 0} \delta^{a 8}, A=$ const.. As mentioned above, this background field acts like a color-chemical potential $\mu_{8} \equiv g A$ for quarks. 
In order to solve the Yang-Mills equation (18), we consider the source terms on the right-hand side. Note first that the contribution from the last term in Eq. (18) is absent in the mean-field approximation for $\Gamma_{2}$, Eq. (13). Second, by dimensional arguments the first term in Eq. (18), which corresponds to the gluon tadpole contribution, is of order $\sim T^{3}$, and thus can be neglected for the temperature range of interest for color superconductivity, $T \sim \phi \sim \mu \exp (-1 / g) \ll \mu$ in weak coupling. Therefore, we only need to consider the quark tadpole contribution which, on account of Eq. (9), takes the form

$$
\mathcal{T}_{a}^{\mu} \equiv-\frac{1}{2} \frac{\delta}{\delta A_{\mu}^{a}(X)} \int_{Y, Z} \operatorname{Tr}\left[S_{0}^{-1}(Y, Z) S(Z, Y)\right]=-\frac{g}{2} \operatorname{Tr}\left[\Gamma_{a}^{\mu} S(0)\right]
$$

where the trace only runs over Nambu-Gor'kov, color, flavor, and Dirac indices. We used the fact that, for translationally invariant systems, $S(X, Y) \equiv S(X-Y)$, i.e., $S(X, X) \equiv S(0)$. The tadpole contribution is simply a constant source term in the Yang-Mills equation (18) for the gluon field.

Taking $A_{\mu}^{a} \equiv 0$ in the quark propagator $S(0)$, the tadpole contribution (27) has been computed for a two-flavor color superconductor in Ref. [9], with the result (5). Let us try to find a solution of the Yang-Mills equation (18), with the expression (5) as a constant source term on the right-hand side. It is obvious that the solution cannot be $A_{\mu}^{a}=0$ for all $a=1, \ldots, 8$. The perturbative calculation of the tadpole performed in Ref. [9], with the result (5), is therefore not consistent with the Yang-Mills equation (18). For a self-consistent solution, we have to allow for a non-zero expectation value of the gluon field $A_{\mu}^{a}$ in the full quark propagator $S(0)$. We have to repeat the calculation of the tadpole with this non-zero background field, before we plug the result back into the Yang-Mills equation (18).

Let us first make a few general remarks concerning the structure of the expectation value $A_{\mu}^{a}$. First, since we consider translationally invariant systems, it has to be constant, $A_{\mu}^{a}=$ const. $\neq 0$. Furthermore, rotational symmetry requires $A_{\mu}^{a} \equiv g_{\mu 0} A^{a}$. Denoting $\mathcal{T}_{a}^{0} \equiv \mathcal{T}_{a}$, we conclude

$$
-g^{2} f_{a b c} f^{b d e} A^{c} A_{d} A_{e}=\mathcal{T}_{a} \equiv 0
$$

by the antisymmetry of the structure constants. Therefore, the expectation value of the gluon field in the quark propagator has to have precisely the magnitude to cancel the quark tadpole contribution.

The calculation of the tadpole contribution including a non-zero $A_{\mu}^{a}=g_{\mu 0} A^{a}$ is completely analogous to the one performed in Ref. [9] for $A_{\mu}^{a}=0$. The only difference is that one has to modify the expressions for the propagators of quasiparticle excitations with fundamental colors 1 and $2, G^{ \pm}(Q)$ (Eq. (21) of Ref. [9]), and for those of unpaired quarks with fundamental color $3, G_{0}^{ \pm}(Q)$ (Eq. (22) of Ref. [9]). If we do not specify the color direction $a$ of the gluon field, this is a non-trivial task, but we may simplify the calculation considerably by the following argument. Note first that, when $A^{a} \equiv 0$ for all colors $a=1, \ldots, 8$, only the eighth color component of the tadpole is non-vanishing, cf. Eq. (5). Hence, we do not need to introduce non-zero gluon fields for $a=1, \ldots, 7$; the corresponding tadpole contributions in these directions in adjoint color space vanish already when $A^{a}=0$ for $a=1, \ldots, 7$. Obviously, we only need a non-vanishing component of the gluon field in the eighth color direction, $A^{a}=A \delta^{a 8}, A \neq 0$, which has to be adjusted such that $\mathcal{T}_{8} \equiv 0$.

As already mentioned in the introduction and after Eq. (9), a non-zero gluon field in the time direction, $A_{\mu}^{a}=A g_{\mu 0} \delta^{a 8}$, acts like a color-chemical potential $\mu_{8} \equiv g A$, which shifts the original quark number chemical potential $\mu$. For quarks of fundamental colors 1 and 2 (which form Cooper pairs in a two-flavor color superconductor) this shift is

$$
\mu \rightarrow \mu+g A_{0}^{8}\left(T_{8}\right)_{11} \equiv \mu+g A_{0}^{8}\left(T_{8}\right)_{22}=\mu+\frac{g A}{2 \sqrt{3}} \equiv \mu_{1}
$$

where we used $\left(T_{8}\right)_{11}=\left(T_{8}\right)_{22}=1 /(2 \sqrt{3})$, and for quarks of fundamental color 3 (which remain unpaired in a two-flavor color superconductor) it is

$$
\mu \rightarrow \mu+g A_{0}^{8}\left(T_{8}\right)_{33}=\mu-\frac{g A}{\sqrt{3}} \equiv \mu_{2},
$$


where we used $\left(T_{8}\right)_{33}=-1 / \sqrt{3}$. In the evaluation of the tadpole contribution by Gerhold and Rebhan 9] we now have to replace $\mu$ by $\mu_{1}$ in their Eq. (21) for the propagator $G^{ \pm}(Q)$ (which describes the propagation of quasiparticles with fundamental colors 1 and 2),

$$
G^{ \pm}(Q)=\sum_{e= \pm} \frac{q_{0} \mp\left(\mu_{1}-e q\right)}{q_{0}^{2}-\left(\mu_{1}-e q\right)^{2}-\left|\phi^{e}\right|^{2}} \Lambda^{ \pm e}(\mathbf{q}) \gamma_{0}
$$

Likewise, we have to replace $\mu$ by $\mu_{2}$ in their Eq. (22) for the propagator $G_{0}^{ \pm}$(which describes the propagation of unpaired quarks with fundamental color 3),

$$
G_{0}^{ \pm}(Q)=\sum_{e= \pm} \frac{q_{0} \mp\left(\mu_{2}-e q\right)}{q_{0}^{2}-\left(\mu_{2}-e q\right)^{2}} \Lambda^{ \pm e}(\mathbf{q}) \gamma_{0}
$$

Then, their calculation can be copied up to Eq. (24), which now reads

$$
\mathcal{T}_{8}=\frac{g}{\pi^{2}}\left(T_{8}\right)_{33} \int_{0}^{\infty} \mathrm{d} q q^{2}\left(\frac{\mu_{1}-q}{\sqrt{\left(\mu_{1}-q\right)^{2}+\phi^{2}}}-\frac{\mu_{2}-q}{\left|\mu_{2}-q\right|}\right) .
$$

(We take the gap function $\phi$ to be real-valued.) Some care has to be taken in the evaluation of this integral. The difference in the chemical potentials, $\delta \mu \equiv \mu_{2}-\mu_{1} \equiv-\sqrt{3} g A / 2$ has to be of the right magnitude to achieve the cancellation of the tadpole, $\mathcal{T}_{8} \equiv 0$. As will be shown below, $\delta \mu \sim \phi^{2} /(g \mu)$, i.e., it is parametrically much smaller than the gap. For the following calculation, it is therefore permissible to make the assumption $\delta \mu \ll \phi$, which is consistent with our final result. We split the integral into three regions, (I) $0 \leq q<\mu_{1}-\delta$, (II) $\mu_{1}-\delta \leq q \leq \mu_{1}+\delta$, and (III) $\mu_{1}+\delta<q<\infty$. The quantity $\delta$ is chosen such that the gap function $\phi$, which is known to rapidly vanish away from the Fermi surface [10], can be neglected in regions (I) and (III). We do not need to specify the magnitude of $\delta$, it suffices to know that $\phi \ll \delta \ll \mu$. We may then write

$$
\begin{aligned}
\mathcal{T}_{8} \simeq \frac{g}{\pi^{2}}\left(T_{8}\right)_{33} & \left\{\int_{\mu_{1}-\delta}^{\mu_{1}+\delta} \mathrm{d} q q^{2}\left(\frac{\mu_{1}-q}{\sqrt{\left(\mu_{1}-q\right)^{2}+\phi^{2}}}-\frac{\mu_{2}-q}{\left|\mu_{2}-q\right|}\right)\right. \\
+ & {\left.\left[\int_{0}^{\mu_{1}-\delta}+\int_{\mu_{1}+\delta}^{\infty}\right] \mathrm{d} q q^{2}\left[\operatorname{sign}\left(\mu_{1}-q\right)-\operatorname{sign}\left(\mu_{2}-q\right)\right]\right\} . }
\end{aligned}
$$

Since $\delta \gg \phi \gg \delta \mu$, the second integral always vanishes. The remaining integral may be evaluated by substituting $\xi \equiv q-\mu_{1}$. The integral over the first term in the integrand is

$$
-\int_{-\delta}^{\delta} \mathrm{d} \xi\left(\mu_{1}+\xi\right)^{2} \frac{\xi}{\sqrt{\xi^{2}+\phi^{2}}} \simeq-2 \mu_{1}\left(\delta^{2}-\phi^{2} \ln \frac{2 \delta}{\phi}\right),
$$

where we only kept the leading and subleading terms and assumed the gap function to be constant over the range of integration. The integral over the second term is best evaluated by shifting the integration variable $\xi \rightarrow \xi^{\prime}=\xi-\delta \mu$

$$
\int_{-\delta-\delta \mu}^{\delta-\delta \mu} \mathrm{d} \xi^{\prime}\left(\mu_{2}+\xi^{\prime}\right)^{2} \operatorname{sign} \xi^{\prime} \simeq 2 \mu_{2}\left(\delta^{2}-\mu_{2} \delta \mu\right)
$$

where we also only kept the leading and subleading terms. Inserting the results (355) and (136) into Eq. (34) we obtain

$$
\mathcal{T}_{8} \simeq \frac{2 g}{\pi^{2}}\left(T_{8}\right)_{33}\left[\mu_{1} \phi^{2} \ln \frac{2 \delta}{\phi}-\delta \mu\left(\mu_{2}^{2}-\delta^{2}\right)\right] \simeq \frac{2 g}{\pi^{2}}\left(T_{8}\right)_{33} \mu^{2}\left(\frac{\phi^{2}}{\mu} \ln \frac{2 \delta}{\phi}-\delta \mu\right)
$$


where in the last step we approximated $\mu_{1} \simeq \mu_{2} \simeq \mu$ and neglected higher-order terms, $\delta^{2} \ll \mu^{2}$. To leading order, we may also assume that $2 \delta / \phi \simeq \exp \left[3 \pi^{2} /(\sqrt{2} g)\right]$ [10]. Consequently, the tadpole vanishes if

$$
\delta \mu \equiv-\frac{\sqrt{3}}{2} g A \simeq \frac{3 \pi^{2}}{\sqrt{2}} \frac{\phi^{2}}{g \mu}[1+O(g)] .
$$

This results justifies our original assumption $\delta \mu \ll \phi$. The expectation value of the gluon field necessary to ensure a vanishing tadpole contribution is thus

$$
A_{\mu}^{8} \equiv A g_{\mu 0} \delta^{a 8} \quad, \quad A \simeq-\sqrt{6} \pi^{2} \frac{\phi^{2}}{g^{2} \mu}[1+O(g)] .
$$

As claimed above, this gives rise to a chemical potential $\mu_{8} \equiv g A$ of precisely the magnitude (6). In a self-consistent calculation, it arises from a non-vanishing expectation value (39) of the gluon field $A_{\mu}^{a}$, which has precisely the magnitude to cancel the tadpole. Let us mention that this "tadpole cancellation" is a well-known mechanism in quantum field theories with spontaneously broken symmetries: upon resummation, perturbatively non-vanishing tadpole contributions like (5) induce non-vanishing expectation values for one-point functions which ultimately cancel the tadpoles. Note also that $A<0$, so that the Fermi surface for red and green quarks is reduced, cf. Eq. (29), while the one for blue quarks is increased, cf. Eq. (30). As argued in the introduction, this works in a way to ensure color neutrality, cf. also next section.

\section{Color neutrality}

Let us compute the color-charge density $n_{a}$ associated with adjoint color $a$. To this end, introduce an explicit color-chemical potential $\mu_{a}$ into the inverse tree-level quark propagator (9). This amounts to adding a term $\mu_{a} \Gamma_{a}^{0}$ to $S_{0}^{-1}$, where $\Gamma_{a}^{0}$ is given by Eq. (10). The thermodynamic pressure is $p \equiv(T / V) \Gamma^{*}$, where $\Gamma^{*}$ is the effective action (11), evaluated at the stationary point defined by Eqs. (14) - (17). The color-charge density $n_{a}$ is then given by

$$
n_{a} \equiv \frac{\partial p}{\partial \mu_{a}}=\frac{1}{2} \frac{T}{V} \int_{X, Y} \operatorname{Tr}\left[S(X, Y) \frac{\partial S_{0}^{-1}(Y, X)}{\partial \mu_{a}}\right]=\frac{1}{2} \operatorname{Tr}\left[\Gamma_{a}^{0} S(0)\right],
$$

where the traces run only over Nambu-Gor'kov, color, flavor, and Dirac indices. Other terms cancel after making use of Eqs. (19) and (20). Finally, we set $\mu_{a}=0$ to compute the color-charge density for vanishing color-chemical potential. If this were non-zero, color superconductivity would break the color gauge symmetry not only spontaneously, but also explicitly by generating a (net) color charge $n_{a}$. This must not be the case. Comparing Eqs. (40) and (27), we obtain the identity

$$
n_{a} \equiv-\frac{1}{g} \mathcal{T}_{a}^{0} \equiv-\frac{1}{g} \mathcal{T}_{a}
$$

The cancellation of the tadpole contribution, $\mathcal{T}_{a}=0$, by a non-vanishing expectation value $A_{\mu}^{a}=A g_{\mu 0} \delta^{a 8}$ for the gluon field, as demonstrated in Sec. 4, thus also ensures color neutrality. This result also implies that, in QCD, color is not broken explicitly by the condensation of Cooper pairs.

\section{Acknowledgement}

D.D.D. acknowledges support by GSI Darmstadt, BMBF, and DAAD. D.H.R. thanks J. Bowers and T. Schäfer for discussions, as well as A. Gerhold, A. Rebhan, I. Shovkovy, and Q. Wang for a critical reading of the manuscript. He also acknowledges support by the INT Seattle where part of this work was done. 


\section{References}

[1] D.J. Gross and F. Wilczek, Phys. Rev. Lett. 30 (1973) 1343 ; H.D. Politzer, Phys. Rev. Lett. 30 (1973) 1346

[2] D. Bailin and A. Love, Phys. Rep. 107 (1984) 325 ; M.G. Alford, K. Rajagopal, and F. Wilczek, Phys. Lett. B 422 (1998) 247 ; R. Rapp, T. Schäfer, E. Shuryak, and M. Velkovsky, Phys. Rev. Lett. 81 (1998) 53

[3] J.R. Schrieffer, Theory of Superconductivity (New York, W.A. Benjamin, 1964); A.L. Fetter and J.D. Walecka, Quantum Theory of Many-Particle Systems (McGraw-Hill, New York, 1971); A.A. Abrikosov, L.P. Gorkov, and I.E. Dzyaloshinski, Methods of Quantum Field Theory in Statistical Physics (Dover, New York, 1963)

[4] see, for instance, the review articles: K. Rajagopal and F. Wilczek, hep-ph/0011333 M. Alford, Annu. Rev. Nucl. Part. Sci. 51 (2001) 131

[5] D.H. Rischke, nucl-th/0305030

[6] R.D. Pisarski and D.H. Rischke, Phys. Rev. D 60 (1999) 094013

[7] M.G. Alford and K. Rajagopal, JHEP 0206 (2002) 031 ; A.W. Steiner, S. Reddy, and M. Prakash, Phys. Rev. D 66 (2002) 094007 ; F. Neumann, M. Buballa, and M. Oertel, Nucl. Phys. A 714 (2003) 481 ; I.A. Shovkovy and M. Huang, Phys. Lett. B 564 (2003) 205 ; M. Huang and I.A. Shovkovy, Nucl. Phys. A 729 (2003) 835

[8] S.B. Rüster and D.H. Rischke, nucl-th/0309022 (to be appear in Phys. Rev. D)

[9] A. Gerhold and A. Rebhan, Phys. Rev. D 68 (2003) 011502

[10] D.T. Son, Phys. Rev. D 59 (1999) 094019 ; T. Schäfer and F. Wilczek, Phys. Rev. D 60 (1999) 114033 ; R.D. Pisarski and D.H. Rischke, Phys. Rev. D 61 (2000) 051501, 074017 ; D.K. Hong, V.A. Miransky, I.A. Shovkovy, and L.C.R. Wijewardhana, Phys. Rev. D 61 (2000) 056001 , Erratum ibid. D 62 (2000) 059903

[11] J.M. Cornwall, R. Jackiw, and E. Tomboulis, Phys. Rev. D 10 (1974) 2428

[12] M.G. Alford, K. Rajagopal, and F. Wilczek, Nucl. Phys. B 537 (1999) 443

[13] A. Kryjevski, Phys. Rev. D 68 (2003) 074008

[14] H. Abuki, hep-ph/0306074

[15] D.H. Rischke, Phys. Rev. D 64 (2001) 094003 ; D.H. Rischke and I.A. Shovkovy, Phys. Rev. D 66 (2002) 054019

[16] D.H. Rischke, Phys. Rev. D 62 (2000) 054017

[17] T. Schäfer, Phys. Rev. D 62 (2000) 094007 ; A. Schmitt, Q. Wang, and D.H. Rischke, Phys. Rev. D 66 (2002) 114010 ; M.G. Alford, J.A. Bowers, J.M. Cheyne, and G.A. Cowan, Phys. Rev. D 67 (2003) 054018

[18] A. Schmitt, Q. Wang, and D.H. Rischke, nucl-th/0301090 (to appear in Phys. Rev. Lett.), nucl-th/0311006 